\section{Review: lithium reduces the risk of suicide compared with placebo in people with depression and bipolar disorder}

doi:10.1136/eb-2013-101493

\section{QUESTION}

Question: Does lithium prevent suicide and self-harm in people with unipolar depression and bipolar disorder?

Outcomes: Suicide, deliberate self-harm (DSH) and allcause mortality. DSH was defined as a non-fatal outcome following a deliberately initiated behaviour (such as selfcutting) or ingestion of a toxic substance or object with the intention of causing self-harm, irrespective of motivation. Suicidal ideation alone was not considered as an outcome.

\section{METHODS}

Design: Systematic review and meta-analysis.

Data sources: MEDLINE, EMBASE, CINAHL, PsycINFO and CENTRAL were searched up to January 2013. This was supplemented by hand search of trial registries for published, unpublished and ongoing clinical trials, and contact with study authors and principal manufacturers.

Study selection and analysis: Randomised controlled trials (RCTs) comparing lithium with placebo or with other drugs used in the long-term treatment ( $\geq 12$ weeks) of mood disorders (diagnosed according to DSM criteria). Eligible studies could include participants of any age or gender; investigate combinations of drugs from the same class or augmentation of treatment with a drug from a different class; and investigate fixed or flexible-dose treatment. Studies including participants with serious medical comorbidity were excluded. OR were calculated using Peto's method. The $\mathrm{I}^{2}$ statistic and visual inspection of forest plots were used to assess heterogeneity. Sensitivity analysis was performed including only people with unipolar depression.

\section{MAIN RESULTS}

Forty-eight RCTs $(\mathrm{n}=6674)$ met inclusion criteria, which compared lithium with placebo (23 studies) or with 14 different active treatment comparators. Twelve RCTs included people with unipolar depression, 19 included people with bipolar disorder and the remaining 17 included people with either condition or with schizoaffective disorder. Mean duration of treatment was 19.1 months. When compared with placebo, lithium reduced the risk of suicide (OR 0.13, 95\% CI 0.03 to 0.66 ) and risk of death from any cause (OR 0.38 , $95 \%$ CI 0.15 to 0.95 ), but had no significant effect on the risk of DSH (OR $0.60,95 \% 0.27$ to 1.32). There was no significant difference between lithium and any active comparator examined for risk of suicide or death from any cause. The only significant effect compared with active treatment was that lithium reduced risk of DSH compared with carbamazepine (OR 0.14, 95\% CI 0.02 to 0.83, 2 RCTs). Subanalyses in people with unipolar depression found that lithium reduced the risk of suicide (OR $0.13,95 \%$ CI 0.02 to 0.76 ) and death from any cause (OR $0.36,95 \% \mathrm{CI} 0.13$ to 0.98) compared with placebo, but had no effect on DSH.

\section{CONCLUSIONS}

Lithium reduces the risk of suicide and overall death compared with placebo in people with unipolar depression or bipolar disorder receiving long-term treatment. Lithium was found to be no more effective than other pharmacological treatments at preventing these outcomes. However, trials comparing lithium with other active treatments were heterogenous, including a wide number of comparators.

\section{ABSTRACTED FROM}

Cipriani A. Lithium in the prevention of suicide in mood disorders: updated systematic review and meta-analysis. BMJ 2013;346:f346.

Correspondence to: Andrea Cipriani, Department of Public Health and Community Medicine, Section of Psychiatry, University of Verona, Verona, Italy; Andrea.cipriani@ psych.ox.ac.uk

Sources of funding: None.
$\mathrm{M}$ ood disorders are a significant cause of excess premature mortality, partly because of the markedly increased risk of suicide, and a leading cause of disability. Evidence-based treatments which reduce the death toll from suicide in these disorders are urgently needed. Lithium has long been associated with a reduction in suicide related to affective disorder. ${ }^{1}$ The early evidence base supporting the use of lithium had many critics. This was understandable given the questionable methodology of many of the early trials. The rise of evidence-based medicine has seen more rigorous lithium trials and evidence which few now dispute. ${ }^{2}$ The review by Cipriani and colleagues adds to this body of work and shows that lithium seems to reduce the risk of death and suicide by more than $60 \%$ compared with a placebo. Lithium may exert its antisuicidal effects by reducing relapse of mood disorder (raising the possibility that other treatments might also share these benefits). The authors recommend that additional mechanisms should also be considered, because there is some evidence that lithium decreases aggression, and possibly impulsivity, which might also mediate the antisuicidal effect.

As with any such review, the findings rest on the quality of the constituent trials, which are imperfect and lack adequate data about active comparators. The generalisability of these findings is also unclear; it would depend on clinical practice improving considerably. Will this evidence change clinical practice? Recent commentators have remarked that, even though the supporting evidence has increased, the use of lithium has declined. ${ }^{3}$ The reasons for this are undoubtedly complex, but must be resisted; with very few evidence-based 'antisuicidal' treatments available, we cannot afford to ignore those we do have.

\section{Allan H Young}

Institute of Psychiatry, King's College London, London, UK

Competing interests None.

\section{REFERENCES}

1. Barraclough B. Suicide prevention, recurrent affective disorder and lithium. Br J Psychiatry 1972;121:391-2.

2. Geddes JR, Burgess S, Hawton K, et al. Long-term lithium therapy for bipolar disorder: systematic review and meta-analysis of randomized controlled trials. Am J Psychiatry 2004;161:217-22.

3. Young $\mathbf{A H}$, Hammond JM. Lithium in mood disorders: increasing evidence base, declining use? Br J Psychiatry 2007;191:474-6. 